Mathematical Medicine and Biology Page 1 of 13

doi:10.1093/imammb/dqnxxx

\title{
The Usage of a Three Compartment Model to Investigate the Metabolic Differences between Hepatic Reductase Null and Wild Type Mice
}

\author{
Lydia Hill ${ }^{1,2}$ Mark A.J. Chaplain ${ }^{1}$ Roland WOlF $^{3}$ YURY KaPElyukH $^{3}$ \\ ${ }^{1}$ Mathematics Division, University of Dundee, \\ Dundee, DDI 4HN, UK. \\ ${ }^{2}$ Applied Physics Group (APHY), Vrije Universiteit Brussel, \\ Pleinlaan 2, Brussels, Belgium. \\ ${ }^{3}$ Division of Cancer Research, Medical Research Institute, Jacqui Wood Cancer Centre \\ University of Dundee, Ninewells Hospital And Medical School \\ Dundee DD1 9SY, UK
}

[Received on 25 January 2013]

\begin{abstract}
The Cytochrome P450 (CYP) system is involved in 90\% of the human bodys interactions with xenobiotics and due to this it has become an area of avid research including the creation of transgenic mice. This paper proposes a three compartment model which is used to explain the drug metabolism in the Hepatic Reductase Null (HRN) mouse developed by the University of Dundee (Henderson et al., 2003).

The model is compared with a two compartment model using experimental data from studies using wild type and HRN mice. This comparison allowed for metabolic differences between the two types of mice to be isolated. The three sets of drug data (Gefitinib, Midazolam and Thalidomide) showed that the transgenic mouse has a decreased rate of metabolism.
\end{abstract}

Keywords: Cytochrome P450 enzymes, Compartment models, Hepatic Reductase Null mice, Drug metabolism.

\section{Introduction}

Over recent years the Cytochrome P450 enzymatic system has become a focus of drug metabolism studies. Since it is involved in 90\% of the human body's interactions with xenobiotics (Parikh et al., 1997) it is an area of interpatient variability in drug response. Due to this transgenic mice have been developed in order to investigate this superfamily of enzymes. One of these mice is the Hepatic Reductase Null (HRN) mouse developed by CXR Biosciences. The three compartment model proposed in this paper was developed to compare the metabolism within a wild type and HRN mouse in order to pinpoint specific differences in mechanism of drug action.

HRN mice are missing the hepatic Cytochrome P450 system but bred so that they are still viable and fertile. These mice are useful as they give one the opportunity to study the metabolism pathways taken by the drug. Through this method it is also possible to gain an insight as to whether the main metabolism of a drug happens in the liver, gastro-intestinal tract, extrahepatically etc. An unexpected consequence of deleting hepatic Cytochrome P450 reductase was the discovery that the P450 content of the liver was increased by approximately a factor of five. These mice help investigate the toxicokinetics of compounds which can show the side effects associated with a drug. This is especially useful when the drug has a narrow therapeutic window, for example, anti-cancer drugs (Henderson et al., 2006). Cyclophosphamide has been pharmacokinetically examined using the Cytochrome P450 reductase null mouse (Pass et al., 
2005).

In pharmacokinetics there are a number of different approaches to modelling drug data including "whole body" approaches, Physiologically Based Pharmacokinetics (PBPK) and compartment models. All three models are based on the representation of the drug absorption using a series of blocks or compartments. The "Whole Body" models have been of great interest since this approach gives a better idea of where the metabolism takes place if all systems are taken into account (Lüpfert and Reichel, 2005). As such compartments are assigned to the lungs, heart, liver, kidney etc. and pharmacokinetic parameters for each are found experimentally or assigned arbitrarily. Flynn et al. (1996) used a Physiologically Based Pharmacokinetic (PBPK) model with a number of compartments in order to analyse ethanol metabolism in mice after an intraperitoneal injection. This approach uses mass balance equations to track both parent and metabolite compounds over time per compartment. This allows a greater insight into the effect of certain organ systems on ethanol concentration. This approach is still used within this area of research although better parameter estimates are still needed (Ramchandani et al., 2001).

Modelling drug data using compartments allows for complex systems to be analysed in a simpler way. The compartment models used in this paper have two and three compartments and were developed from the basis of a one block model. A paper by Yu et al. (1999) used a one-compartment model for the metabolites in their experiments. They were investigating Cyclophosphamide and Ifosfamide in rats with reference to Cytochrome P450 catalysed metabolism with respect to phenbarbital pre-treatment. These drugs were given as intraperitoneal injections but since they were modelling the metabolites, the one compartment approach is equivalent to a two compartment approach since there is a phase of metabolism prior to the data used.

In Klein et al. (2012) a two compartmental model was used while investigating the effect of the CYP2C9 enzyme on Warfarin metabolism. The article was focussed on the prevalence of Drug-Drug Interactions (DDI) which can arise in Asian populations due to polymorphisms in this enzyme.

Grass (1997) used a two compartment STELLA (Structural Thinking Experimental Learning Laboratory with Animation) model in order to analyse the drugs ketorolac and ganciclovir. This model is a physiologically based formulation used to describe dynamics in the gastro-intestinal tract. The parameters were simulated from in vitro data including animal cultures and used to predict oral drug absorption in humans.

An example of a three compartment model was shown in Sugano et al. (2011) where it was used to represent an epithelial cell and parameterised using membrane permeabilities. This article allowed for pharmacokinetic parameters to be estimated from the data which can aid with drug design.

A three compartment model was applied to mouse data in Shen et al. (2011) where the link between 5-methoxy-N,N-dimethyltryptamine metabolism and the CYP2D6 enzyme was studied. Their model describe a nonlinear elimination from the central compartment based on Michaelis-Menten kinetics and the peripheral compartment is solely to represent the CYP2D6 dependent metabolism.

A review of modelling techniques including oral administrations with respect to metal metabolism is outlined in Curis et al. (2009). This paper outlines the advantages and disadvantages of different models as well as the need for "dummy" compartments that have no strict use as they represent the absorbed from and excreted to compartments. The paper presents the mammalian model for humans, which is generally a compartment representing blood or plasma with peripheral sections for organ systems that are relevant to the drug in question.

Doan and Boje (2000) analyse several different pharmacokinetic models with reference to the endogenous inhibitors and their effect on drug half-lives and concentration. They investigate this by using compartment models and different administration techniques for the inhibitor. This includes oral and 
bolus with the oral ingestion of food, which contain the inhibitor. The parameters in this case were found using data from literature sources from experiments on rats which were given aspartame.

The oral administration of CHS 828, a cancer agent on rats, was analysed by Friberg et al. (2005) using a one-compartment model. The study investigated the effect of change of therapeutic schedule on drug efficacy on breast cancer cell lines.

Techniques for retrieving parameter values are usually done in vitro with some more recent studies doing experiments in vivo (Lombardo et al., 2002) as well since converting parameters (Baranczewski et al., 2006) from one to the other have been unreliable (Chiu et al., 2007). Other methods include trying to find parameters by reproducing a verified set of data (Curis et al., 2009). The other problem for this experimental data is that sometimes the particular animal used is not a good representative of the modelled animal. For example, using rat data to predict parameters for a human model (Harris and Barton, 2008) may be partially similar in mechanism in the body but the differences could cause errors in the estimation and therefore a better understanding is needed to account for these.

The focus of this article is to introduce the three compartment model and through its usage investigate the difference between the metabolism of the wild type and HRN mouse. Within section 2 we introduce the drugs analysed and explain the development of the model. In the results section (section 3 ) the model fit and the fitted parameters are analysed to give information on whether the three compartment model has the best fit and how the two mice differ with respect to parameters.

\section{Mathematical Methods}

Compartment modelling is widely used in pharmacokinetics, as it is a means of reducing complexity in drug metabolism problems. The simplest model is one with a single well-mixed compartment, which is used mostly to describe intravenous administrations since transfer into the blood system is assumed to be $100 \%$. As such there is no need for an absorption phase to be taken into account such as there would be with oral or intraperitoneal administrations. This is due to the need for the oral drug to be absorbed through the Gastro-Intestinal (GI) tract membranes (Smith et al., 2001) and the intraperitoneal injection needs to pass out of the peritoneal membrane surrounding the abdominal cavity.

The experimental data provided by CXR Biosciences were taken from procedures on both Hepatic Reductase Null (HRN) and wild type mice (three of each). The drugs investigated here were given through intraperitoneal injection. An intraperitoneal injection is used in experiments on small rodents since they are very small and so have limited muscle mass and small veins (Fox et al., 2006). As such it is easier to inject into the peritoneum since intravenous and intramuscular administrations are not so possible. Two different compartment models were applied to the sets of drug data as shown in Figure 1.

\subsection{Specific Drugs}

There were three sets of drug data and these were for Gefitinib, Midazolam and Thalidomide. These were chosen due to the fact that there is only one cytochrome P450 enzyme responsible for the metabolism. What follows is an explanation of the properties and mechanism of action for each drug.

2.1.1 Gefitinib This drug is an epidermal growth factor receptor (EGFR) inhibitor and as such is used to treat many human cancers (Chang et al., 2008). In lung and breast cancers EGFR is overexpressed and this can lead to uncontrolled cell proliferation and this is why this drug is important in cancer treatment. This drug is metabolised by CYP3A4 (Scripture et al., 2005), which means this must take place in the liver and small intestine since this is where this enzyme can be found. The substance was injected into 
the peritoneum with a dose of $5 \mathrm{mg} / \mathrm{kg}$.

2.1.2 Midazolam This drug is a short-acting hypnotic-sedative drug that is commonly used in dentistry, endoscopy and in combination with local anaesthesia (Wishart et al., 2006). It is frequently used in palliative care as a sedative or anticonvulsant (Morita et al., 2003). It is supposed to be used over short periods of time with the longest time of use reported being 35 days. It is a benzodiazepine that acts as a central nervous system depressant and as such has pharmacodynamic properties including amnesia and sedation (Wishart et al., 2006). It increases (gamma)-amino butyric acid (GABA) activity, which causes a calming effect resulting in sleep. This drug is metabolised by CYP3A4 (Lewis, 2000) that is found in the liver and small intestine. As with Gefitinib this is given by intraperitoneal injection of $1 \mathrm{mg} / \mathrm{kg}$.

2.1.3 Thalidomide This compound was initially used as a non-barbiturate hypnotic but it had to be removed from distribution due to teratogenic effects. It is currently used for inflammatory and immunological disorders as it shows immunosuppressive activity (Wishart et al., 2006). In addition to this it shows anti-angiogenic activity, which might mean it can be used as part of cancer therapy. Although many of the effects of the drug have been documented there is still a lot more information about its mechanism of action required (Strasser and Ludwig, 2002). Recently there has been research with zebrafish in Japan that has isolated the protein that caused the birth defects in the children of Thalidomide patients (Ito et al., 2010). The Cytochrome P450 enzyme responsible for Thalidomide metabolism is $2 \mathrm{C} 19$ (Ando et al., 2002) and this is found in the liver and is subject to polymorphisms. This drug was given by intraperitoneal injection with a dose of $20 \mathrm{mg} / \mathrm{kg}$.

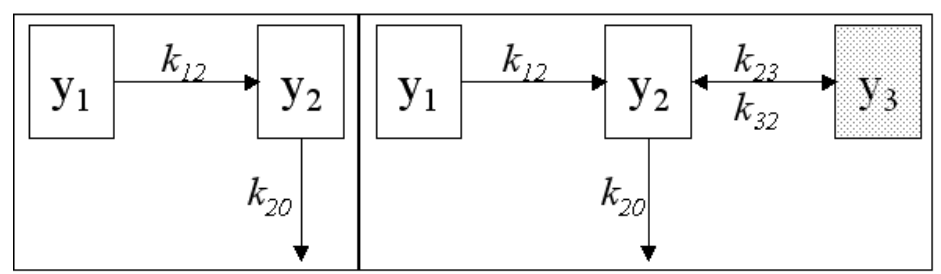

FIG. 1. Schematic diagram of the models. The two compartment model (within the bordered area) and the proposed three compartment model.

\subsection{Two Compartment Model}

The two compartments shown in Figure 1 allow extravascular administration (e.g. intraperitoneal) to be modelled since the drug can start at the injection/dose site $\left(y_{1}\right)$ and pass through to the bloodstream $\left(y_{2}\right)$. As such there should be an absorption phase into the bloodstream and then an elimination/excretion curve.

Using the Law of Mass Action, the ordinary differential equations for the above model are:

$$
\begin{aligned}
& \frac{d y_{1}}{d t}=-k_{12} y_{1} \\
& \frac{d y_{2}}{d t}=k_{12} y_{1}-k_{20} y_{2}
\end{aligned}
$$


Within this model $y_{1}$ and $y_{2}$ represent the primary dosing site (e.g. peritoneum in intraperitoneal administration) and the bloodstream respectively. The raw data is taken to represent the $y_{2}$ concentration. The parameters for this model are fitted using nonlinear least squares algorithm with the raw data and the analytical solutions are shown in the Appendix.

\subsection{Three Compartment Model}

This model further extends the previous one through an extra compartment. The configuration and ordinary differential equations for this model are as follows:

$$
\begin{aligned}
& \frac{d y_{1}}{d t}=-k_{12} y_{1} \\
& \frac{d y_{2}}{d t}=k_{12} y_{1}-k_{23} y_{2}+k_{32} y_{3}-k_{20} y_{2} \\
& \frac{d y_{3}}{d t}=k_{23} y_{2}-k_{32} y_{3}
\end{aligned}
$$

In this model the first and second compartments represent the same as in the previous model i.e. $y_{2}$ represents the bloodstream and $y_{1}$ the injection/dose site. The bloodstream $y_{2}$ is assumed to only have positive values as this is physiologically realistic. As we can see there is a rate of absorption into the main compartment $\left(y_{2}\right)$ indicated by $k_{12}$. From the main compartment there are two ways for the drug to be distributed - it can be passed into compartment three or be expelled from the system entirely. The expulsion rate from the main compartment is $k_{20}$. Compartment three represents lipid in the liver i.e. $k_{23}$ is the rate at which the drug is stored and $k_{32}$ is the rate at which it is released back into the system. The current assumption is that $k_{23}>>k_{32}$ i.e. that it is easier to get into compartment three than it is to leave.

As with the two compartment model the parameters were fitted using nonlinear least squares and the analytical solutions are given in the Appendix.

\section{Results}

For each drug the model parameters are compared and contrasted with the Wild Type (WT) and HRN mouse. These parameters can be found in table 1 and table 2. The plots (figures 2, 3 and 4) show the average drug data (over three mice) and the fitted model. The fit was assessed using the Akaikes Information Criterion (AIC) and a F test (tables 3 and 4) on each average data set compared with the each of the fitted models. The AIC statistic was used with the F statistic since the models are nested and it uses a penalty for too many parameters.

\subsection{Gefitinib}

The parameters for Gefitinib shown in tables 1 and 2 indicate that for most of the parameters (with the exception of $y_{1}(0)$ and $k_{32}$ for the two and three Compartment models respectively) the transgenic mouse shows slower rate of metabolism than that of the wild type mouse. In figure 2 both the two and three compartment models fit the data well although the bimodal nature of the HRN data lends itself more to the three compartment model. The AIC values suggest that the best model for the HRN mouse 


\begin{tabular}{|c|c|c|c|c|}
\hline \multicolumn{2}{|c|}{ Two Compartment } & WT & HRN & Ratio (3dp) \\
\hline \multirow{3}{*}{ Gefitinib } & $y_{1}(0)$ & 55331.46 & 67711.24 & $1: 1.224$ \\
& $k_{12}$ & 0.447 & 0.141 & $1: 0.316$ \\
& $k_{20}$ & 15.679 & 11.290 & $1: 0.720$ \\
\hline \multirow{3}{*}{ Midazolam } & $y_{1}(0)$ & 835.445 & 2242.681 & $1: 2.684$ \\
& $k_{12}$ & 1.376 & 0.581 & $1: 0.422$ \\
& $k_{20}$ & 8.777 & 6.272 & $1: 0.715$ \\
\hline \multirow{3}{*}{ Thalidomide } & $y_{1}(0)$ & 77565.25 & 24408.21 & $1: 3.793$ \\
& $k_{12}$ & 0.561 & 2.126 & $1: 0.072$ \\
& $k_{20}$ & 3.908 & 0.283 & $1: 0.315$ \\
\hline
\end{tabular}

Table 1. Gefitinib, Midazolam and Thalidomide Model Parameters for two compartment model.

\begin{tabular}{|c|c|c|c|c|}
\hline \multicolumn{2}{|c|}{ Three Compartment } & WT & HRN & Ratio (3dp) \\
\hline \multirow{5}{*}{ Gefitinib } & $y_{1}(0)$ & 3278.265 & 1583.595 & $1: 0.483$ \\
& $k_{12}$ & 4.953 & 4.443 & $1: 0.897$ \\
& $k_{23}$ & 2.691 & 2.190 & $1: 0.814$ \\
& $k_{32}$ & 1.784 & 2.136 & $1: 1.197$ \\
& $k_{20}$ & 0.749 & 0.224 & $1: 0.300$ \\
\hline \multirow{5}{*}{ Midazolam } & $y_{1}(0)$ & 234.059 & 278.353 & $1: 1.189$ \\
& $k_{12}$ & 4.263 & 4.501 & $1: 1.056$ \\
& $k_{23}$ & 1.697 & 1.026 & $1: 0.605$ \\
& $k_{32}$ & 1.091 & 3.264 & $1: 2.991$ \\
& $k_{20}$ & 1.983 & 0.771 & $1: 0.389$ \\
\hline \multirow{5}{*}{ Thalidomide } & $y_{1}(0)$ & 82347.45 & 122055.7 & $1: 1.482$ \\
& $k_{12}$ & 0.610 & 0.437 & $1: 0.717$ \\
& $k_{23}$ & 1.677 & 0.667 & $1: 0.398$ \\
& $k_{32}$ & 5.449 & 0.718 & $1: 0.132$ \\
& $k_{20}$ & 4.209 & 0.351 & $1: 0.351$ \\
\hline
\end{tabular}

Table 2. Gefitinib, Midazolam and Thalidomide Model Parameters for three compartment model.

\begin{tabular}{|c|c|c|c|c|c|c|}
\hline \multirow{2}{*}{ Data } & \multicolumn{3}{|c|}{ Two Compartment } & \multicolumn{3}{c|}{ Three Compartment } \\
\cline { 2 - 7 } & Gefitinib & Midazolam & Thalidomide & Gefitinib & Midazolam & Thalidomide \\
\hline HRN & $\mathbf{8 4 . 2 7 1}$ & $\mathbf{1 9 . 4 4 0}$ & $\mathbf{9 9 . 0 4 7}$ & 100.874 & 75.439 & 155.047 \\
\hline WT & 92.288 & $\mathbf{2 1 . 7 4 8}$ & $\mathbf{8 4 . 2 0 0}$ & $\mathbf{8 8 . 4 3 8}$ & 77.748 & 140.200 \\
\hline
\end{tabular}

Table 3. AIC (Akaike's Information Criterion) for Gefitinib, Midazolam and Thalidomide. The model chosen for each data set is indicated in bold.

is the two compartment model whereas for the WT this is the three compartment model. The average data for the HRN mouse shows at least two peaks, which are not features of either of the compartment models considered. The $\mathrm{F}$ test results suggest that the three compartment model provides a better fit for the data even though it has more parameters. 


\begin{tabular}{|c|c|c|c|}
\hline \multirow{2}{*}{ Data } & \multicolumn{3}{|c|}{ F test } \\
\cline { 2 - 4 } & Gefitinib & Midazolam & Thalidomide \\
\hline HRN & $3.177 e^{-10}$ & 0.934 & 0.001 \\
\hline WT & $3.953 e^{-11}$ & 0.481 & 0.002 \\
\hline
\end{tabular}

Table 4. F statistic values for Gefitinib, Midazolam and Thalidomide.
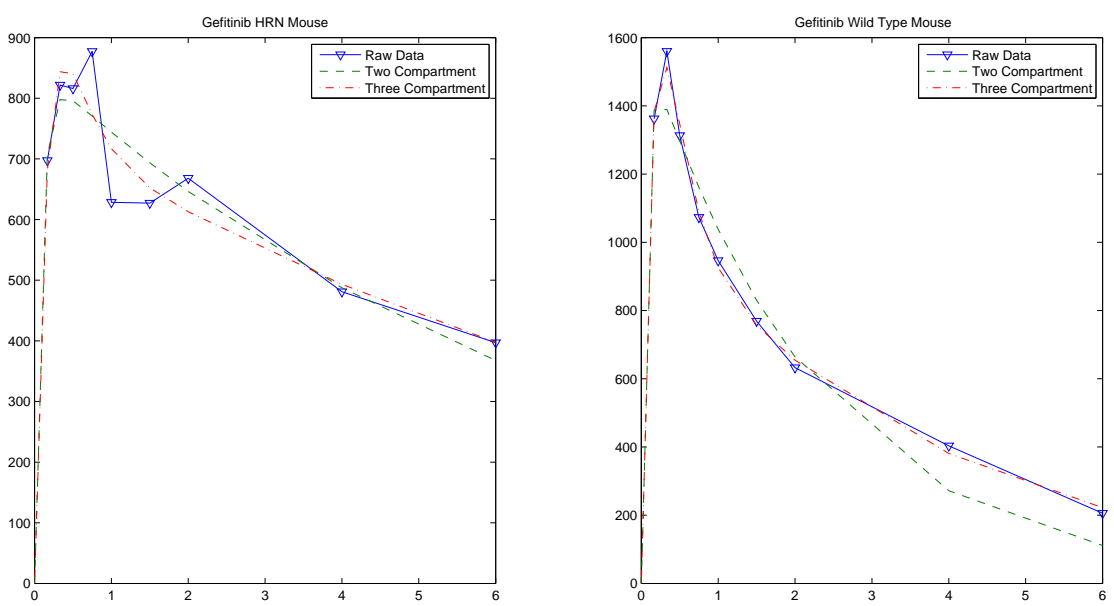

FIG. 2. (Colour online) Plots showing the Gefitinib drug concentration against time for the raw data $(\nabla)$ and computational simulation results from HRN (left) and WT (right).

\subsection{Midazolam}

The parameters for the two models for this drug are shown in tables 1 and 2. For the Midazolam models the fitted initial concentrations and the $k_{12}$ and $k_{32}$ in the 3 compartment model are all larger in the transgenic mice than the wild type. However for all the other rate parameters the rates (excretion and transfer) are slower in the HRN mouse. Both models fit the absorption and excretion phase fine but after the 2-hour point the two compartment fitted model does not fit well as shown in figure 3. According to the AIC values (table 3) the two compartment model is the best for the data sets and the F-test supports this outcome.

\subsection{Thalidomide}

For this drug the model parameters are listed in tables 1 and 2. As with Midazolam all the initial concentrations for the models are larger for the transgenic mouse than for the wild type. However for the rate parameter all of the rates are reduced in the HRN mouse. The fit of these models to these data points is good but the peaks are not similar in shape as shown in Figure 4. The AIC values suggest that the best model for the data is the two Compartment model but the F-test favours the three compartment model. 

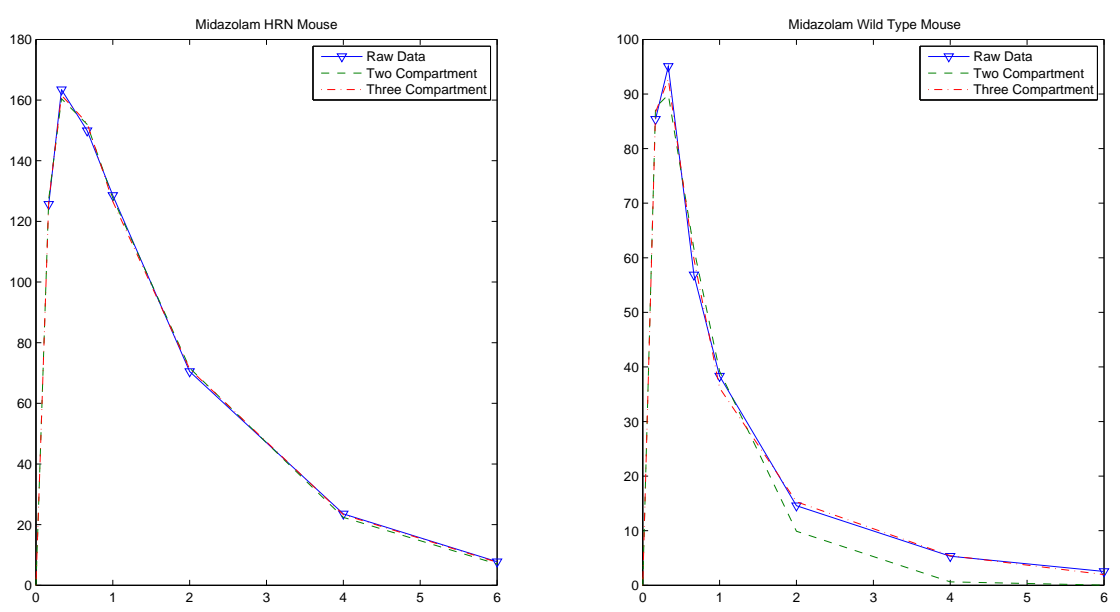

FIG. 3. (Colour online) Plots showing the Midazolam drug concentration against time for the raw data $(\nabla)$ and computational simulation results from HRN (left) and WT (right).
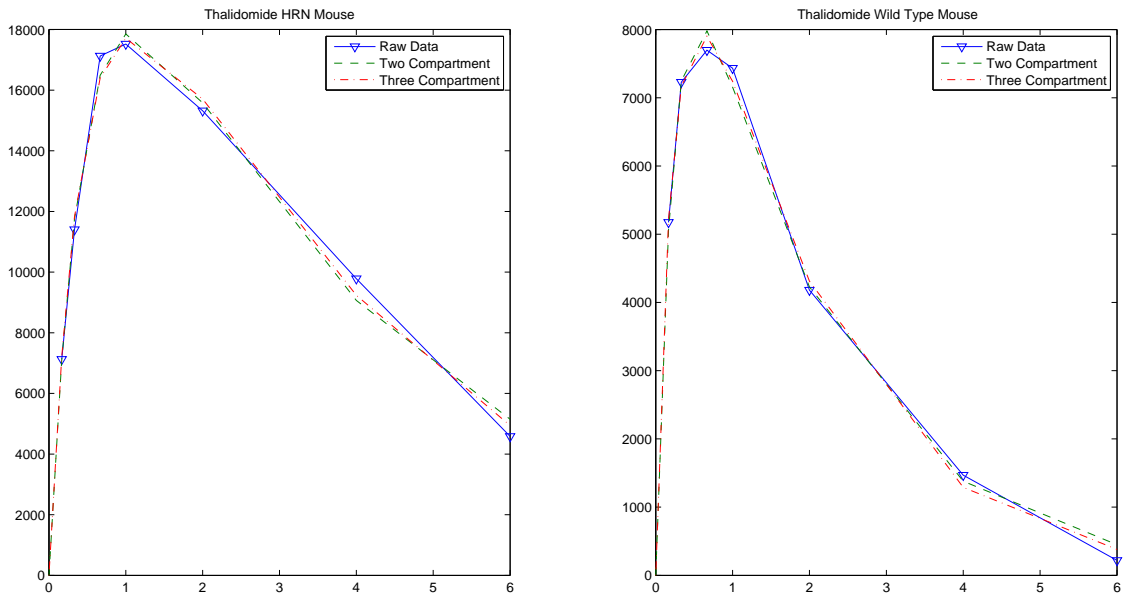

FIG. 4. (Colour online) Plots showing the Thalidomide drug concentration against time for the raw data $(\nabla)$ and computational simulation results from HRN (left) and WT (right).

\section{Discussion}

Throughout this paper the same two models have been fitted to the data sets with varying amount of success. A table summarising the success in fitting the models to the CXR Bioscience data is shown in table 5. It shows that although the two compartment model fits well to the data sometimes a more 


\begin{tabular}{|c|c|c|c|c|}
\hline \multirow{2}{*}{ Drug } & \multicolumn{2}{|c|}{ Wild Type } & \multicolumn{2}{c|}{ Hepatic Reductase Null } \\
\cline { 2 - 5 } & F test & AIC & F test & AIC \\
\hline Gefitinib & 3 & 3 & 3 & 2 \\
\hline Midazolam & 2 & 2 & 2 & 2 \\
\hline Thalidomide & 3 & 2 & 3 & 2 \\
\hline
\end{tabular}

Table 5. Summary of Compartmental Models chosen for each CXR Bioscience Experiment.

complex model is necessary. This is shown particularly in Midazolam where the data can be fitted using the two compartment model but for Gefitinib and Thalidomide the data requires the more complex three compartment model. The need for extra complexity could stem from a physiological difference or the necessity for multiple time scales due to multiple Cytochrome P450 enzymes acting on the drug or the drug needing to move area to get metabolised. The novel three compartment model outlined in this paper takes a step towards addressing this need for more complexity.

When the parameters for the HRN and wild type mice were compared for most of the data sets the HRN rates were slower than their counterpart. This could mean there is a metabolic difference between the two mice stemming from the genetic knockout. Since metabolism is dependent on a number of factors it is difficult to pin this down to one physiological parameter.

The parameters for Gefitinib shown in tables 1 and 2 indicate that for most of the parameters (with the exception of $k_{32}$ for Three Compartment model) the transgenic mouse shows slower rate of metabolism than that of the wild type mouse. This fact indicates that compartment 3 in the HRN mouse is less like a "fatty liver" as was intended since it is easier to leave than enter.

For the Midazolam models the fitted initial concentrations, $k_{12}$ and $k_{32}$ in the 3 compartment model are all larger in the transgenic mice than the wild type. However for all the other rate parameters the rates (excretion and transfer) are slower in the HRN mouse. Both models fit the absorption and excretion phases fine but after the 2-hour point the fitted 2 compartment model does not fit well. Out of the two models the three compartment shows the best visual fit to the data sets.

For Thalidomide all the initial concentrations for the models are larger for the transgenic mouse than for the wild type.

Although the initial concentration for the two compartment model was roughly four times in the HRN mouse the associated rate parameters were both less than a third of the wild type rate. This suggests that physiologically the absorption and elimination of the transgenic mouse was much slower than a normal mouse metabolising Thalidomide. The visual fit of the two and three compartment model to the data sets was good with the exception of the peak area.

The problem with fitting the compartment models to these sets of data is generated by experimental constraints. It would be easier if more data were available instead of just three mice of each type for each drug. This would mean that the average would be statistically more representative which would aid in providing a better fit. Other than this constraint it would have been useful if more samples could have been taken or taken more regularly. However this is unfeasible since the volume of blood in a mouse is finite and takes a while to replenish. It is due to these constraints that sampling is prioritised within the first hour after the dose.

Since both models were fitted using the non-linear least squares in Microsoft Excel the results are comparable. This area of modelling is useful since the curves generated are able to mimic real life. If it was possible to fully explain the action of the drug for both types of mouse it would be easier to show the specific metabolic differences between the transgenic and wild type. From the work presented it can 
be seen that the HRN mouse has a slower metabolism than the wild type.

\section{Acknowledgements}

The authors gratefully acknowledge the support of EPSRC (case studentship) and CXR Biosciences. L.H. is currently funded by the Research Foundation Flanders (FWO) and the Belgian Science Policy Office under Grant No. IAP-VI/10.

\section{Appendix: Analytical Solutions}

The solution to the two compartment model is obtained through straightforward integration and is given as:

$$
\begin{aligned}
& y_{1}(t)=y_{1}(0) e^{-k_{12} t} \\
& y_{2}(t)=\frac{k_{12} y_{1}(0) e^{-k_{20} t}}{k_{12}-k_{20}}-\frac{k_{12} y_{1}(0) e^{-k_{12} t}}{k_{12}-k_{20}} .
\end{aligned}
$$

The solution to the three compartment model, while a little unwieldy, may be obtained using MAPLE, and with the substitutions $F=\left[k_{20}^{2}-2 k_{32} k_{20}+2 k_{20} k_{23}+k_{32}^{2}+2 k_{32} k_{23}+k_{23}^{2}\right]^{1 / 2}$ and $G=\left[k_{23}^{2}+\left(2 k_{20}+2 k_{32}\right) k_{23}+\left(k_{32}-k_{20}\right)^{2}\right]^{1 / 2}$ is given as: 


$$
\begin{aligned}
& y_{1}(t)=y_{1}(0) e^{-k_{12} t} \\
& y_{2}(t)=-\left(\left(-4\left(k_{20}^{2}-k_{32} k 20+2 k_{20} k_{23}+k_{32}^{2}+2 k_{32} k_{23}+k_{23}^{2}\right)^{1 / 2} k_{20}+4 F k_{32}-4 F k_{23}-4 k_{20}^{2}\right.\right. \\
& \left.+8 k_{32} k_{20}-8 k_{20} k_{23}-4 k_{32}^{2}-8 k_{32} k_{23}-4 k_{23}^{2}\right) k_{12}^{2}+\left(4 F k_{20}^{2}+\left(8 F k_{23}+4 k_{20}^{2}-8 k_{32} k_{20}+8 k_{20} k_{23}\right.\right. \\
& \left.+4 k_{32}^{2}+8 k_{32} k_{23}+4 k_{23}^{2}\right) k_{20}-4 F k_{32}^{2}+\left(4 k_{20}^{2}-8 k_{32} k_{20}+8 k_{20} k_{23}+4 k_{32} h 2+8 k_{32} k_{23}+4 k_{23}^{2}\right) k_{32} \\
& \left.+4 F k_{23}^{2}+\left(4 k_{20}^{2}-8 k_{32} k_{20}+8 k_{20} k_{23}+4 k_{32}^{2}+8 k_{32} k_{23}+4 k_{23}^{2}\right) k_{23}\right) k_{12}+\left(-4 F k_{32}-k_{20}^{2}+2 k_{32} k_{20}\right. \\
& \left.-2 k_{20} k_{23}-k_{32}^{2}-2 k_{32} k_{23}-k_{23}^{2}\right) k_{20}^{2}+\left(4 F k_{32}^{2}+\left(-4 F k_{23}-2 k_{20}^{2}+4 k_{32} k_{20}-4 k_{20} k_{23}-2 k_{32}^{2}\right.\right. \\
& \left.\left.-4 k_{32} k_{23}-2 k_{23}^{2}\right) k_{32}+\left(-2 k_{20}^{2}+4 k_{32} k_{20}-4 k_{20} k_{23}-2 k_{32}^{2}-4 k_{32} k_{23}-2 k_{23}^{2}\right) k_{23}\right) k_{20}+\left(-k_{20}^{2}\right. \\
& \left.+2 k_{32} k_{20}-2 k_{20} k_{23}-k_{32}^{2}-2 k_{32} k_{23}-k_{23}^{2}\right) k_{32}^{2}+\left(-2 k_{20}^{2}+4 k_{32} k_{20}-4 k_{20} k_{23}-2 k_{32}^{2}-4 k_{32} k_{23}\right. \\
& \left.-2 k_{23}^{2}\right) k_{23} k_{32}+\left(-k_{20}^{2}+2 k_{32} k_{20}-2 k_{20} k_{23}-k_{32}^{2}-2 k_{32} k_{23}-k_{23}^{2}\right) k_{23}^{2}+\left(k_{20}^{2}-2 k_{32} k_{20}+2 k_{20} k_{23}\right. \\
& \left.\left.+k_{32}^{2}+2 k_{32} k_{23}+k_{23}^{2}\right)^{2}\right) k_{12} y_{1}(0) e^{\left(\left(-0.5\left(k_{20}-k_{32}-k_{23}-F\right) t\right)\right.}\left(\left(( - 2 k _ { 1 2 } + k _ { 2 0 } + k _ { 3 2 } + k _ { 2 3 } - F ) \left(-2 k_{12}\right.\right.\right. \\
& \left.+k_{20}+k_{32}+k_{23}+F\right)\left(k_{20}^{2}-2 k_{32} k_{20}+2 k_{20} k_{23}+k_{32}^{2}+2 k_{32} k_{23}+k_{23}^{2}\right)\left(2 k_{12}-k_{20}-k_{32}-k_{23}\right. \\
& -F)))^{-1}+\left(\left(-4 F k_{20}+4 F k_{32}-4 F k_{23}+4 k_{20}^{2}-8 k_{32} k_{20}+8 k_{20} k_{23}+4 k_{32}^{2}+8 k_{32} k_{23}+4 k_{23}^{2}\right) k_{12}^{2}\right. \\
& +\left(4 F k_{20}^{2}+\left(8 F k_{23}-4 k_{20}^{2}+8 k_{32} k_{20}-8 k_{20} k_{23}-4 k_{32}^{2}-8 k_{32} k_{23}-4 k_{23}^{2}\right) k_{20}-4 F k_{32}^{2}+\left(-4 k_{20}^{2}\right.\right. \\
& \left.+8 k_{32} k_{20}-8 k_{20} k_{23}-4 k_{32}^{2}-8 k_{32} k_{23}-4 k_{23}^{2}\right) k_{32}+4 F k_{23}^{2}+\left(-4 k_{20}^{2}+8 k_{32} k_{20}-8 k_{20} k_{23}-4 k_{32}^{2}\right. \\
& \left.\left.-8 k_{32} k_{23}-4 k_{23}^{2}\right) k_{23}\right) k_{12}+\left(-4 F k_{32}+k_{20}^{2}-2 k_{32} k_{20}+2 k_{20} k_{23}+k_{32}^{2}+2 k_{32} k_{23}+k_{23}^{2}\right) k_{20}^{2} \\
& +\left(4 F k_{32}^{2}+\left(-4 F k_{23}+2 k_{20}^{2}-4 k_{32} k_{20}+4 k_{20} k_{23}+2 k_{32}^{2}+4 k_{32} k_{23}+2 k_{23}^{2}\right) k_{32}+\left(2 k_{20}^{2}-4 k_{32} k_{20}\right.\right. \\
& \left.\left.+4 k_{20} k_{23}+2 k_{32}^{2}+4 k_{32} k_{23}+2 k_{23}^{2}\right) k_{23}\right) k_{20}+\left(k_{20}^{2}-2 k_{32} k_{20}+2 k_{20} k_{23}+k_{32}^{2}+2 k_{32} k_{23}+k_{23}^{2}\right) k_{32}^{2} \\
& +\left(2 k_{20}^{2}-4 k_{32} k_{20}+4 k_{20} k_{23}+2 k_{32}^{2}+4 k_{32} k_{23}+2 k_{23}^{2}\right) k_{23} k_{32}+\left(k_{20}^{2}-2 k_{32} k_{20}+2 k_{20} k_{23}+k_{32}^{2}\right. \\
& \left.+2 k_{32} k_{23}+k_{23}^{2}\right) k_{23}^{2}-\left(\left(k_{20}^{2}-2 k_{32} k_{20}+2 k_{20} k_{23}+k_{32}^{2}+2 k_{32} k_{23}+k_{23}^{2}\right)^{2}\right) k_{12} y_{1}(0) \\
& \left.e^{\left(\left(-0.5\left(k_{20}-k_{32}-k_{23}+F\right) t\right)\right.}\right)\left(\left(( - 2 k _ { 1 2 } + k _ { 2 0 } + k _ { 3 2 } + k _ { 2 3 } - F ) ( - 2 k _ { 1 2 } + k _ { 2 0 } + k _ { 3 2 } + k _ { 2 3 } + F ) \left(k_{20}^{2}\right.\right.\right. \\
& \left.\left.\left.-2 k_{32} k_{20}+2 k_{20} k_{23}+k_{32}^{2}+2 k_{32} k_{23}+k_{23}^{2}\right)\left(2 k_{12}-k_{20}-k_{32}-k_{23}+F\right)\right)\right)^{-1} \\
& +\frac{(0.5)\left(-8 e^{\left(-k_{12} t\right)} F k_{23} k_{12}^{2}+8 e^{\left(-k_{12} t\right)} F k_{23} k_{32} k_{12}\right) y_{1}(0)}{\left(\left(-2 k_{12}+k_{20}+k_{32}+k_{23}-F\right)\left(-2 k_{12}+k_{20}+k_{32}+k_{23}+F\right) F k_{23}\right)}, \\
& y_{3}(t)=2 \frac{e^{\left(-0.5\left(k_{20}-k_{32}-k_{23}+F\right)\right) t} k_{23} k_{12} y_{1}(0)}{F\left(2 k_{12}-k_{20}-k_{32}-k_{23}+F\right)}-2 \frac{e^{\left(-0.5\left(k_{20}-k_{32}-k_{23}-F\right)\right) t} k_{23} k_{12} y_{1}(0)}{F\left(2 k_{12}-k_{20}-k_{32}-k_{23}-F\right)} \\
& -\frac{\left(-2 y_{1}(0) e^{-k_{12} t+\left(\left(2\left(k_{20}+k_{32}+k_{23}-F\right)\right)^{-1}\right) t} e^{\left(\left(2\left(k_{20}+k_{32}+k_{23}+G\right)\right)^{-1}\right) t}\right.}{-2 k_{12}+k_{20}+k_{32}+k_{23}-F} \\
& +\frac{2 y_{1}(0) G e^{-k_{12} t+\left(\left(2\left(k_{20}+k_{32}+k_{23}+F\right)\right)^{-1}\right) t} e^{\left(\left(2\left(k_{20}+k_{32}+k_{23}-G\right)\right)^{-1}\right) t}}{\left.\left(-2 k_{12}+k_{20}+k_{32}+k_{23}+F\right)\right) k_{23} k_{12}\left(e^{-t\left(k_{20}+k_{32}+k_{23}\right)}\right)} \text {. }
\end{aligned}
$$




\section{References}

Ando, Y., Fuse, E., Figg, W. D., Jun 2002. Thalidomide metabolism by the cyp2c subfamily. Clinical Cancer Research 8 (6), 1964-1973.

Baranczewski, P., Stańczak, A., Sundberg, K., Svensson, R., Wallin, A., Jansson, J., Garberg, P., Postlind, H., 2006. Introduction to in vitro estimation of metabolic stability and drug interactions of new chemical entities in drug discovery and development. Pharmacological Reports 58, 453-472.

Chang, G.-C., Yu, C.-T. R., Tsai, C.-H., Tsai, J.-R., Chen, J.-C., Wu, C.-C., Wu, W.-J., Hsu, S.-L., 2008. An epidermal growth factor inhibitor, gefitinib, induces apoptosis through a p53-dependent upregulation of pro-apoptotic molecules and downregulation of anti-apoptotic molecules in human lung adenocarcinoma a549 cells. European Journal of Pharmacology 600, 37-44.

Chiu, W. A., Barton, H. A., DeWoskin, R. S., Schlosser, P., Thompson, C. M., Sonawane, B., Lipscomb, J. C., Krishnan, K., 2007. Evaluation of physiologically based pharmacokinetic models for use in risk assessment. Journal of Applied Toxicology 27, 218-237.

Curis, E., Nicolis, I., Bensaci, J., Deschamps, P., Bénazeth, S., 2009. Mathematical modeling in metal metabolism: overview and perspectives. Biochimie 91, 1238-1254.

Doan, K. M. M., Boje, K. M., 2000. Theoretical pharmacokinetic and pharmacodynamic simulations of drug delivery mediated by blood-brain barrier transporters. Biopharmaceutics \& Drug Disposition $21,261-278$.

Flynn, E., Pastino, G., Sultatos, L., 1996. Development and application of a physiologically based pharmacokinetic model for ethanol in the mouse. Alcohol \& Alcoholism 31 (4), 365-374.

Fox, J., Barthold, S., Davisson, M., Newcomer, C., Quimby, F., Smith, A. (Eds.), 2006. The Mouse in Biomedical Research: Normative Biology, Husbandry, and Models. Academic Press.

Friberg, L. E., Hassan, S. B., Lindhagen, E., Larsson, R., Karlsson, M. O., 2005. Pharmacokineticpharmacodynamic modelling of the schedule-dependent effect of the anti-cancer agent chs 828 in a rat hollow fibre model. European Journal of Pharmaceutical Sciences 25, 163-173.

Grass, G., 1997. Simulation models to predict oral drug absorption. Advanced Drug Delivery Review 23, 199-219.

Harris, L. A., Barton, H. A., 2008. Comparing single and repeated dosimetry data for perfluorooctane sulfonate in rats. Toxicology Letters 181, 148-156.

Henderson, C. J., Otto, D. M. E., Carrie, D., Magnuson, M. A., McLaren, A. W., Rosewell, I., Wolf, C. R., Apr 2003. Inactivation of the hepatic cytochrome p450 system by conditional deletion of hepatic cytochrome p450 reductase. J Biol Chem 278 (15), 13480-13486.

URL http://dx.doi.org/10.1074/jbc.M212087200

Henderson, C. J., Pass, G. J., Wolf, C. R., 2006. The hepatic cytochrome p450 reductase null mouse as a tool to identify a successful candidate entity. Toxicology Letters 162, 111-117.

Ito, T., Ando, H., Suzuki, T., Ogura, T., Hotta, K., Imamura, Y., Yamaguchi, Y., Handa, H., 2010. Identification of a primary target of thalidomide teratogenicity. Science 327, 1345-1350. 
Klein, K., Gueorguieva, I., Aarons, L., Apr 2012. Population pharmacokinetic modelling of s-warfarin to evaluate the design of drug-drug interaction studies for cyp2c9. J Pharmacokinet Pharmacodyn 39 (2), 147-160.

Lewis, D., 2000. On the recognition of mammalian microsomal cytochrome p450 substrates and their characteristics. Biochemical Pharmacology 60, 293306.

Lombardo, F., Obach, R. S., Shalaeva, M. Y., Gao, F., 2002. Prediction of volume of distribution values in humans for neutral and basic drugs using physicochemical measurements and plasma protein binding data. Journal of Medicinal Chemistry 45, 2867-2876.

Lüpfert, C., Reichel, A., 2005. Development and application of physiologically based pharmacokineticmodeling tools to support drug discovery. Chemistry \& Biodiversity 2, 1462-1486.

Morita, T., Tei, Y., Inoue, S., 2003. Correlation of the dose of midazolam for symptom control with administration periods: the possibility of tolerance. Journal of Pain and Symptom Management 25, 369-375.

Parikh, A., Gillam, E. M., Guengerich, F. P., 1997. Drug metabolism by escherichia coli expressing human cytochromes p450. Nature Biotechnology 15, 784-788.

Pass, G. J., Carrie, D., Boylan, M., Lorimore, S., Wright, E., Houston, B., Henderson, C. J., Wolf, C. R., 2005. Role of hepatic cytochrome p450s in the pharmacokinetics and toxicity of cyclophosphamide: studies with the hepatic cytochrome p450 reductase null mouse. Cancer Research 65, 4211-4217.

Ramchandani, V. A., Bosron, W. F., Li, T. K., 2001. Research advances in ethanol metabolism. Pathologie Biologie 49, 676-682.

Scripture, C. D., Sparreboom, A., Figg, W. D., 2005. Modulation of cytochrome p450 activity: implications for cancer therapy. Lancet Oncology 6, 780-789.

Shen, H.-W., Jiang, X.-L., Yu, A.-M., Jul 2011. Nonlinear pharmacokinetics of 5-methoxy-n,ndimethyltryptamine in mice. Drug Metab Dispos 39 (7), 1227-1234.

Smith, D., van de Waterbeemd, H., Walker, D., 2001. Pharmacokinetics and Metabolism in Drug Design. Wiley.

Strasser, K., Ludwig, H., 2002. Thalidomide treatment in multiple myeloma. Blood Reviews 16, $207-$ 215.

Sugano, K., Shirasaka, Y., Yamashita, S., Oct 2011. Estimation of michaelis-menten constant of efflux transporter considering asymmetric permeability. Int J Pharm 418 (2), 161-167.

Wishart, D. S., Knox, C., Guo, A. C., Shrivastava, S., Hassanali, M., Stothard, P., Chang, Z., Woolsey, J., 2006. Drugbank: a comprehensive resource for in silico drug discovery and exploration. Nucleic Acids Research 34, D668-D672.

Yu, L. J., Drewes, P., Gustafsson, K., Brain, E. G., Hecht, J. E., Waxman, D. J., 1999. In vivo modulation of alternative pathways of p-450-catalyzed cyclophosphamide metabolism: impact on pharmacokinetics and antitumor activity. Journal of Pharmacology and Experimental Therapeutics 288, 928-937. 